\title{
Brane cosmology with curvature corrections
}

\author{
G. Kofinas ${ }^{1 *}$, R. Maartens ${ }^{2 \dagger}$, E. Papantonopoulos ${ }^{3 \ddagger}$ \\ ${ }^{1}$ Centro de Estudios Científicos, Casilla 1469, Valdivia, Chile \\ ${ }^{2}$ Institute of Cosmology and Gravitation, University of Portsmouth, Portsmouth PO1 2EG, UK and \\ ${ }^{3}$ Department of Physics, National Technical University of Athens, GR 15773 Athens, Greece
}

(Dated: June 26, 2021)

\begin{abstract}
We study the cosmology of the Randall-Sundrum brane-world where the Einstein-Hilbert action is modified by curvature correction terms: a four-dimensional scalar curvature from induced gravity on the brane, and a five-dimensional Gauss-Bonnet curvature term. The combined effect of these curvature corrections to the action removes the infinite-density big bang singularity, although the curvature can still diverge for some parameter values. A radiation brane undergoes accelerated expansion near the minimal scale factor, for a range of parameters. This acceleration is driven by the geometric effects, without an inflaton field or negative pressures. At late times, conventional cosmology is recovered.
\end{abstract}

\section{INTRODUCTION}

The Randall-Sundrum II model [1] provides a simple phenomenology for exploring brane-world gravity and associated ideas from string theory. Matter and gauge interactions are localized on the brane, while gravity accesses the infinite extra dimension, but is localized at low energies due to the warping (curvature) of the extra dimension. The cosmological generalization of the Randall-Sundrum model is characterized by an unconventional evolution at early times, while standard cosmology is recovered at late times [2, 3].

The Randall-Sundrum model is based on the EinsteinHilbert action in five dimensions. This gravitational action can be generalized in various ways. Two important generalizations have been considered recently. The first is a four-dimensional scalar curvature term in the brane action. This induced gravity correction arises because the localized matter fields on the brane, which couple to bulk gravitons, can generate via quantum loops a localized four-dimensional world-volume kinetic term for gravitons [4, 5]. The second is a Gauss-Bonnet correction to the five-dimensional action. This gives the most general action with second-order field equations in five dimensions [6]. Furthermore, in an effective action approach to string theory, the Gauss-Bonnet term corresponds to the leading order quantum corrections to gravity, and its presence guarantees a ghost-free action [7].

The induced gravity model, with no brane tension (i.e., the brane is not self-gravitating) and no bulk cosmological constant, leads to very different behaviour than the Randall-Sundrum model. In the Randall-Sundrum case, gravity becomes 5-dimensional at high energies, so that general relativity is modified in the early universe. By contrast, in the induced gravity case, corrections to gen-

\footnotetext{
*kofinas@cecs.cl

†roy.maartens@port.ac.uk

‡lpapa@central.ntua.gr
}

eral relativity become significant at low energies/ late times, and the early universe evolution agrees with standard general relativity [5, 8]. When the brane tension and bulk cosmological constant are included, the latetime modifications persist, with less fine-tuning needed, but further modifications are introduced [4, 9, 10, 11]. (Astrophysical implications of induced gravity have also been considered [12].)

The Gauss-Bonnet model is like the Randall-Sundrum model in the sense that modifications to general relativity arise in the early universe at high energies. The graviton zero mode is also localized at low energies, as in the Randall-Sundrum case 13. The brane cosmology of the Gauss-Bonnet theory has been investigated in Refs. 14, 15, 16, 17, 18].

Here, we investigate the effects of the combined curvature corrections, from both induced gravity and GaussBonnet. In some sense, these are the leading-order corrections to the gravitational action, and there is no obvious way to argue that one effect is dominant over the other. Indeed, the corrections operate at different energy levels. Induced gravity introduces intriguing late-time modifications, which can accelerate the universe even in the absence of dark energy [8, 11]. However, one expects that string-theory type modifications to the EinsteinHilbert action must also operate at early times, and so it is sensible to incorporate the Gauss-Bonnet correction. Furthermore, there are indications that the induced gravity model may suffer from strong coupling effects at intermediate scales [19]. Adding a Gauss-Bonnet correction may remove or alleviate this problem.

The Gauss-Bonnet correction significantly changes the bulk field equations, whereas the induced gravity correction is 4-dimensional and does not affect the bulk solution away from the brane. The induced gravity effects are felt via the junction conditions at the brane boundary of the $Z_{2}$-symmetric bulk, and lead to modifications in the brane Friedmann equation (in addition to the GaussBonnet modifications).

At early times, the Randall-Sundrum model gives an unconventional cosmology, with the Hubble rate $H$ scal- 
ing as $\rho$, rather than $\rho^{1 / 2}$ as in general relativity. The Gauss-Bonnet correction to this picture changes the $\rho$ dependence of $H$ to $\rho^{2 / 3}$, and therefore an infinite-density big bang is encountered, as in the Randall-Sundrum case. The combined effect of Gauss-Bonnet and induced gravity modifications eliminates the infinite-density solutions, because the scale factor is bounded. However, the initial curvature may diverge since there is a range of parameters for which the solutions start their evolution with infinite acceleration. In the low-energy regime of these solutions, the standard cosmology is recovered (with positive Newton constant).

The paper is organized as follows. In Sec. II, we give the most general action which incorporates the induced gravity and Gauss-Bonnet corrections, and we derive the modified Friedmann equation. In Sec. III, we discuss the cosmology arising from this equation, and Sec. IV gives our conclusions.

\section{FRIEDMANN EQUATION ON THE BRANE}

For convenience and without loss of generality, we can choose the extra-dimensional coordinate $y$ such that the brane is fixed at $y=0$. The total gravitational action is

$$
\begin{aligned}
& S_{\text {grav }}=\frac{1}{2 \kappa_{5}^{2}} \int d^{5} x \sqrt{-{ }^{(5)} g}\left\{{ }^{(5)} R-2 \Lambda_{5}+\alpha\left[{ }^{(5)} R^{2}\right.\right. \\
& \left.\left.-4^{(5)} R_{A B}{ }^{(5)} R^{A B}+{ }^{(5)} R_{A B C D}{ }^{(5)} R^{A B C D}\right]\right\} \\
& +\frac{r}{2 \kappa_{5}^{2}} \int_{y=0} d^{4} x \sqrt{-{ }^{(4)} g}\left[{ }^{(4)} R-2 \Lambda_{4}\right]
\end{aligned}
$$

where the Gauss-Bonnet coupling $\alpha$ has dimensions (length $)^{2}$ and is defined as

$$
\alpha=\frac{1}{8 g_{s}^{2}},
$$

with $g_{s}$ the string energy scale, while the induced-gravity crossover length scale is

$$
r=\frac{\kappa_{5}^{2}}{\kappa_{4}^{2}}=\frac{M_{4}^{2}}{M_{5}^{3}}
$$

Here, the fundamental $\left(M_{5}\right)$ and the four-dimensional $\left(M_{4}\right)$ Planck masses are given by

$$
\kappa_{5}^{2}=8 \pi G_{5}=M_{5}^{-3}, \quad \kappa_{4}^{2}=8 \pi G_{4}=M_{4}^{-2} .
$$

The brane tension is given by

$$
\lambda=\frac{\Lambda_{4}}{\kappa_{4}^{2}},
$$

and is non-negative. (Note that $\Lambda_{4}$ is not the same as the cosmological constant on the brane.)
We assume there are no sources in the bulk other than $\Lambda_{5}$. Varying Eq. (1) with respect to the bulk metric ${ }^{(5)} g_{A B}$, we obtain the field equations:

$$
\begin{aligned}
& { }^{(5)} G_{A B}-\frac{\alpha}{2}\left[{ }^{(5)} R^{2}-4^{(5)} R_{C D}{ }^{(5)} R^{C D}\right. \\
& \left.+{ }^{(5)} R_{C D E F}{ }^{(5)} R^{C D E F}\right]{ }^{(5)} g_{A B} \\
& +2 \alpha\left[{ }^{(5)} R^{(5)} R_{A B}-2^{(5)} R_{A C}{ }^{(5)} R_{B}{ }^{C}\right. \\
& \left.-2^{(5)} R_{A C B D}{ }^{(5)} R^{C D}+{ }^{(5)} R_{A C D E}{ }^{(5)} R_{B}{ }^{C D E}\right] \\
& =-\Lambda_{5}{ }^{(5)} g_{A B}+\kappa_{5}^{2(\text { loc })} T_{A B} \hat{\delta}(y),
\end{aligned}
$$

where ${ }^{(4)} g_{A B}={ }^{(5)} g_{A B}-n_{A} n_{B}$ is the induced metric on the hypersurfaces $\{y=$ constant $\}$, with $n^{A}$ the normal vector. The localized energy-momentum tensor of the brane is

$$
{ }^{(\mathrm{loc})} T_{A B} \equiv{ }^{(4)} T_{A B}-\lambda{ }^{(4)} g_{A B}-\frac{r}{\kappa_{5}^{2}}{ }^{(4)} G_{A B},
$$

and we have used the normalized Dirac delta function, $\hat{\delta}(y)=\sqrt{{ }^{(4)} g /{ }^{(5)} g} \delta(y)$.

The pure Gauss-Bonnet correction is the case $r=0$, the pure induced gravity correction is the case $\alpha=0$, and the Randall-Sundrum case is $r=0=\alpha$.

To determine the Friedmann equation on the brane, one can project the five-dimensional Einstein equations to four dimensions, following the approach of [11, 20]. This is a difficult task because of the presence of the Lovelock tensor. An easier approach is to solve the 5dimensional field equations in suitable coordinates and impose the junction conditions, following the RandallSundrum case [2], as generalized to the Gauss-Bonnet case in Ref. 15].

A homogeneous and isotropic brane at fixed coordinate position $y=0$ in the bulk is given by the five-dimensional line element

$$
\begin{aligned}
{ }^{(5)} d s^{2}= & -N^{2}(t, y) d t^{2}+A^{2}(t, y) \gamma_{i j} d x^{i} d x^{j} \\
& +B^{2}(t, y) d y^{2}
\end{aligned}
$$

where $\gamma_{i j}$ is a constant curvature three-metric, with curvature index $k=0, \pm 1$, and $N(t, 0)=1$, so that $t$ is proper time along the brane. The presence of the fourdimensional curvature scalar in the gravitational action does not affect the bulk equations. If we define

$$
\Phi=\frac{1}{N^{2}} \frac{\dot{A}^{2}}{A^{2}}-\frac{1}{B^{2}} \frac{A^{2}}{A^{2}}+\frac{k}{A^{2}},
$$

then Eq. (6) reduces to [15]

$$
\begin{aligned}
& \frac{\dot{A}}{A} \frac{N^{\prime}}{N}+\frac{A^{\prime}}{A} \frac{\dot{B}}{B}-\frac{\dot{A}^{\prime}}{A}=0, \\
& \Phi+2 \alpha \Phi^{2}=\frac{\Lambda_{5}}{6}+\frac{\mathcal{C}}{A^{4}},
\end{aligned}
$$

where $\mathcal{C}$ is an integration constant, giving the mass of the bulk black hole. (The bulk reduces to Schwarzschild$\operatorname{Ad} S_{5}$ if $\alpha=0$ and to $\operatorname{Ad} S_{5}$ if $\alpha=0=\mathcal{C}$.) 
The solutions of the bulk equations for Eq. (8) [16] fall into two classes: either the fine-tuning $\Lambda_{5}=-3 / 4 \alpha$ holds, or it does not, in which case the unique solution is the black hole solution found and discussed in Ref. 21].

Assuming that the matter on the brane is a perfect fluid, and using the matching conditions for a braneworld in Gauss-Bonnet gravity 22], the jump of the (00) component of Eq. (6) across the brane gives

$$
\begin{gathered}
\frac{2}{r}\left[1+4 \alpha\left(H^{2}+\frac{k}{a^{2}}-\frac{A_{+}^{\prime 2}}{3 a^{2} b^{2}}\right)\right] \frac{A_{+}^{\prime}}{a b} \\
=-\frac{\kappa_{4}^{2}}{3}(\rho+\lambda)+H^{2}+\frac{k}{a^{2}},
\end{gathered}
$$

where $a=A(t, 0), b=B(t, 0), H=\dot{a} / a$ is the Hubble rate, and $2 A_{+}^{\prime}=-2 A_{-}^{\prime}$ is the discontinuity of the first derivative. Equations (9) and (12) imply

$$
\begin{gathered}
\frac{4}{r^{2}}\left[1+\frac{8}{3} \alpha\left(H^{2}+\frac{k}{a^{2}}+\frac{\Phi_{0}}{2}\right)\right]^{2}\left(H^{2}+\frac{k}{a^{2}}-\Phi_{0}\right) \\
=\left[H^{2}+\frac{k}{a^{2}}-\frac{\kappa_{4}^{2}}{3}(\rho+\lambda)\right]^{2},
\end{gathered}
$$

where $\Phi_{0}=\Phi(t, 0)$. This is a cubic equation for $H^{2}+k / a^{2}$, and its real solution will give the Friedmann equation of induced gravity with a Gauss-Bonnet term in the bulk.

In the limit $r \rightarrow 0$, Eq. (13) becomes

$$
\begin{aligned}
{[1} & \left.+\frac{8}{3} \alpha\left(H^{2}+\frac{k}{a^{2}}+\frac{\Phi_{0}}{2}\right)\right]^{2}\left(H^{2}+\frac{k}{a^{2}}-\Phi_{0}\right) \\
& =\frac{\kappa_{5}^{4}}{36}(\rho+\lambda)^{2} .
\end{aligned}
$$

The single real solution of this cubic which is compatible with the $\alpha \rightarrow 0$ limit of Eq. (14), is the Friedmann equation with Gauss-Bonnet correction [16]

$$
H^{2}+\frac{k}{a^{2}}=\frac{1}{8 \alpha}\left(-2+\frac{64 I^{2}}{J}+J\right)
$$

where the dimensionless quantities $I, J$ are given by

$$
\begin{aligned}
& I=\frac{1}{8}\left(1+4 \alpha \Phi_{0}\right)= \pm \frac{1}{8}\left[1+\frac{4}{3} \alpha \Lambda_{5}+\frac{8 \alpha \mathcal{C}}{a^{4}}\right]^{1 / 2} \\
& J=\left[\frac{\kappa_{5}^{2} \sqrt{\alpha}}{\sqrt{2}}(\rho+\lambda)+\sqrt{\frac{\kappa_{5}^{4} \alpha}{2}(\rho+\lambda)^{2}+(8 I)^{3}}\right]^{2 / 3}
\end{aligned}
$$

The negative sign in Eq. (16) does not provide the correct $\alpha \rightarrow 0$ limit.

In the other limit, $\alpha \rightarrow 0$, Eq. (13) yields

$$
\frac{4}{r^{2}}\left(H^{2}+\frac{k}{a^{2}}-\Phi_{0}\right)=\left[H^{2}+\frac{k}{a^{2}}-\frac{\kappa_{4}^{2}}{3}(\rho+\lambda)\right]^{2} .
$$

The solution is the Friedmann equation of the induced gravity model $4,9,10,11]$

$$
\begin{aligned}
& H^{2}+\frac{k}{a^{2}}=\frac{\kappa_{4}^{2}}{3}(\rho+\lambda)+\frac{2}{r^{2}} \\
& \pm \frac{1}{\sqrt{3} r}\left[4 \kappa_{4}^{2}(\rho+\lambda)-2 \Lambda_{5}+\frac{12}{r^{2}}-\frac{12 \mathcal{C}}{a^{4}}\right]^{1 / 2}
\end{aligned}
$$

[The \pm sign is independent of that in Eq. (16).]

Returning to the general case of both curvature corrections, we need the real solution of Eq. (13) in the simplest possible form. We define the dimensionless parameter

$$
\beta=\frac{256 \alpha}{9 r^{2}}
$$

and the dimensionless variables

$$
\begin{aligned}
& P=1+3 \beta I, \\
& Q=\beta\left[\frac{1}{4}+I+\frac{\kappa_{4}^{2} \alpha}{3}(\rho+\lambda)\right], \\
& X=\beta\left[\frac{1}{4}+I+\alpha\left(H^{2}+\frac{k}{a^{2}}\right)\right] .
\end{aligned}
$$

Then, Eq. (13) takes the form

$$
X^{3}-P X^{2}+2 Q X-Q^{2}=0 .
$$

The single real solution of this equation which is compatible with the $\alpha \rightarrow 0$ limit of Eq. (13), i.e. with Eq. (19), is

$$
X=\frac{P}{3}-\frac{2}{3} \sqrt{P^{2}-6 Q} \cos \left(\Theta \pm \frac{\pi}{3}\right),
$$

where

$$
\Theta(P, Q)=\frac{1}{3} \arccos \left[\frac{2 P^{3}+27 Q^{2}-18 P Q}{2\left(P^{2}-6 Q\right)^{3 / 2}}\right] .
$$

This solution corresponds to the positive sign in Eq. (16), while the negative sign does not provide the correct $\alpha \rightarrow$ 0 limit [27]. The \pm sign in Eq. (25) is the same as that in Eq. (19). The region in $(P, Q)$-space for which Eq. (25) is defined, is

$$
\begin{aligned}
& 1 \leq P<\frac{4}{3}, \\
& 2\left[9 P-8-(4-3 P)^{3 / 2}\right] \leq 27 Q \\
& \quad \leq 3 P[3-\sqrt{3(3-2 P)}] .
\end{aligned}
$$

(These conditions imply that $Q \geq 0, X \geq 0,0<\Theta \leq$ $\pi / 6$.)

Finally, we can write the Friedmann equation of the combined Gauss-Bonnet and induced gravity braneworld as

$$
\begin{aligned}
& H^{2}+\frac{k}{a^{2}}=\frac{4-3 \beta}{12 \beta \alpha} \\
& \quad-\frac{2}{3 \beta \alpha} \sqrt{P^{2}-6 Q} \cos \left(\Theta \pm \frac{\pi}{3}\right) .
\end{aligned}
$$


This has a very different structure than its limiting forms, Eqs. (15) and (19). A closed system of equations for the brane-world follows from the jump of the linear equation (10), which gives the conservation law,

$$
\dot{\rho}+3 H \rho(1+w)=0,
$$

where $w=p / \rho \geq-1$ and $\rho \geq 0$. If $w$ is constant, then $\rho=\rho_{0}\left(a_{0} / a\right)^{3(1+w)}$, and we can choose $a_{0}=1$.

In cases where some particular fine-tuning of the form $\alpha \Lambda_{5}=$ constant, or $\alpha \mathcal{C}=$ constant is satisfied, then, as we can directly check, none of the solutions of Eq. (13) gives Eq. (19) in the limit $\alpha \rightarrow 0$. This is actually expected, since under such fine-tunings, the Gauss-Bonnet term cannot be considered as a perturbation of the background theory, and the limit $\alpha \rightarrow 0$ is accompanied by divergences of the bulk vacuum energy $\Lambda_{5}$ or the bulk black hole mass $\mathcal{C}$. However, the brane-world cosmology of these cases is still governed by Eq. (13), with explicit solutions depending on the considered region of $(P, Q)$ space. The fine-tuning $\alpha \Lambda_{5}=-3 / 4$ characterizes the first class of bulk solutions found in Ref. [16]. This is also the case of Chern-Simons gravitational theory, with $1 /\left|\Lambda_{5}\right|$ being the scale of the (A)dS gauge group (see e.g. Ref. 23]), while black hole solutions of the theory have been found in Ref. [24]. We do not discuss these situations further here.

\section{COSMOLOGICAL DYNAMICS}

The dimensionless variable $P$ is a function of $I$ and carries the information of the bulk onto the brane, since by Eq. (16) it depends on the bulk cosmological constant $\Lambda_{5}$ and the mass $\mathcal{C}$ of the bulk black hole. The dimensionless variable $Q$ includes information about the matter and energy content of the brane. These are the key variables determining the cosmological dynamics.

The four-dimensional scalar curvature term of the induced gravity and the Gauss-Bonnet term in the fivedimensional space are all curvature corrections to the Randall-Sundrum model. One could be led to expect that $r^{2}$ and $\alpha$ are of the same order. However, this is not necessarily true. The crossover scale $r$ of the induced gravity appears in loops involving matter particles, and depending on the mass, it can be arbitrarily large. On the other hand, the Gauss-Bonnet coupling $\alpha$ arises from integrating out massive string modes, and depending on the scale of the theory, it can also be arbitrarily large. The dimensionless parameter $\beta$ measures the relative strengths of induced gravity and Gauss-Bonnet corrections. In principle, $\beta$ can take any positive value. However, since the last term of Eq. (29) is non-positive, we have an upper bound on $\beta$ for $k \geq 0$,

$$
\frac{\alpha}{r^{2}} \leq \frac{3}{64} \text { for } k \geq 0
$$

\section{A. No infinite-density big bang}

An important feature arises from inequalities (27) and (28), which show that $P$ and hence $Q$ are bounded from above. Furthermore, Eqs. (21) and (27) show that $I$ is bounded from above (and positive). Therefore, it follows from Eq. (22) that the energy density $\rho$ cannot become infinite, which means that an infinite-density singularity $a=0$ is never encountered:

$$
a(t) \geq a_{0}>0, \quad \rho(t) \leq \rho_{0}<\infty .
$$

This is true independent of the spatial curvature $k$, or the equation of state.

This result is remarkable since the Gauss-Bonnet correction, which is expected to dominate at early times, on its own does not remove the infinite-density singularity [15, 16, 18], while the induced gravity correction on its own mostly affects the late-time evolution. However, the combination of these curvature corrections is effectively "nonlinear", producing a result that is not obviously the superposition of their separate effects. In general terms, the early-universe behaviour is strongly modified by the effective coupling of the 5D curvature to the matter [15]. It is certainly desirable to achieve singularity avoidance via stringy- and quantum-type corrections, since we expect quantum gravity to remove the singularities of general relativity. However, as we show below, the finiteness of the maximum density at the minimal epoch $a_{0}$ may be accompanied by a divergence of the 4D curvature scalar on the brane, depending on the parameters. (Similar behaviour has been previously noted in effective 4D string gravity theories 25].) In this case, there is not full singularity avoidance, since the brane spacetime geometry is undefined in the initial state.

In the pure Gauss-Bonnet theory $(\mathcal{C} \geq 0)$, the earlyuniverse evolves from infinite density at $a=0$. The Friedmann equation (15) for $\mathcal{C}=0$, or for $\mathcal{C}>0, w>0$, is approximated by

$$
H^{2}+\frac{k}{a^{2}} \approx\left(\frac{\kappa_{5}^{2}}{16 \alpha}\right)^{2 / 3} \rho^{2 / 3} .
$$

For $w>0$, or for $\mathcal{C}=0=k$, the density term dominates the curvature term, and

$$
a \approx \text { const } \times t^{1 /(1+w)} .
$$

The Gauss-Bonnet correction causes the universe to expand faster relative to Einstein gravity, for which $a \propto$ $t^{2 / 3(1+w)}$, and to the Randall-Sundrum model, for which $a \propto t^{1 / 3(1+w)}$. At the same time, a given energy density produces a smaller expansion rate in the Gauss-Bonnet case. This means that there is less Hubble friction for a given potential than in general relativity, so that slowroll is more difficult to achieve. For the same reason, scalar perturbations generated during slow-roll inflation will have a smaller amplitude than those generated at the same energy density in general relativity. This is opposite to the Randall-Sundrum model [26]. 


\section{B. Geometric inflation in a radiation universe}

We assume $\mathcal{C}>0$, i.e. there is a black hole present in the bulk. Defining the acceleration variable $f=\ddot{a} / a=$ $\dot{H}+H^{2}$, we obtain from Eqs. (29) and (30) that

$$
\begin{aligned}
f= & \frac{4-3 \beta}{12 \beta \alpha}+\frac{\cos (\Theta \pm \pi / 3)}{3 \beta \alpha \sqrt{P^{2}-6 Q}} \times \\
& \times\left[c_{1}+\sigma(1-3 w)\left|(P-1)^{2}-c_{2}\right|^{3(1+w) / 4}\right. \\
& \left.+\left(P^{2}-6 Q\right) \frac{\dot{\Theta}}{H} \tan \left(\Theta \pm \frac{\pi}{3}\right)\right]
\end{aligned}
$$

where

$$
\begin{aligned}
& \left(P^{2}-6 Q\right) \frac{\dot{\Theta}}{H}= \\
& =\frac{1}{\sqrt{3} \sqrt{4 Q(9 P-8)-4 P^{2}(P-1)-27 Q^{2}}} \times \\
& \times\left\{2(2 P-9 Q)\left[(P-1)^{2}-c_{2}\right]\right. \\
& \quad-3 \sigma(1+w)[3 Q-2 P(P-1)] \times \\
& \left.\quad \times\left|(P-1)^{2}-c_{2}\right|^{3(1+w) / 4}\right\},
\end{aligned}
$$

and

$$
\begin{aligned}
c_{1} & =-2+\beta\left(3+4 \alpha \Lambda_{4}\right)-3 \beta^{2}\left(3+4 \alpha \Lambda_{5}\right) / 32, \\
c_{2} & =\frac{3}{32} \beta^{2}\left(3+4 \alpha \Lambda_{5}\right) \\
\sigma & =\beta \alpha \kappa_{4}^{2} \rho_{0}\left(\frac{8}{9 \beta^{2} \alpha \mathcal{C}}\right)^{3(1+w) / 4} .
\end{aligned}
$$

These equations are formidably complicated, and we do not attempt an exhaustive analysis. Instead, we show that for a radiation brane in the presence of a bulk black hole, there is a range of parameters for which there is inflationary expansion, $f>0$, near $a_{0}$.

For a radiation era, $w=\frac{1}{3}$,

$$
\begin{aligned}
f= & \frac{4-3 \beta}{12 \beta \alpha}+\frac{\cos (\Theta \pm \pi / 3)}{3 \beta \alpha \sqrt{P^{2}-6 Q}} \times \\
& \times\left[c_{1}+\left(P^{2}-6 Q\right) \frac{\dot{\Theta}}{H} \tan \left(\Theta \pm \frac{\pi}{3}\right)\right]
\end{aligned}
$$

where

$$
\begin{aligned}
& \left(P^{2}-6 Q\right) \frac{\dot{\Theta}}{H}=2\left|(P-1)^{2}-c_{2}\right| \times \\
& \quad \times \frac{4 \sigma P^{2}-2(2 \sigma-1) P-3(2 \sigma+3) Q}{\sqrt{3} \sqrt{4 Q(9 P-8)-4 P^{2}(P-1)-27 Q^{2}}} .
\end{aligned}
$$

We assume that

$$
\Lambda_{5}>-\frac{3}{4 \alpha}
$$

and define the additional parameters

$$
\begin{aligned}
P_{1} & =1+\sqrt{\frac{c_{2}}{2}} \\
Q_{1} & =\frac{1}{12}\left(c_{1}+c_{2}+2+2 \sqrt{2 c_{2}}\right) \\
\tau & =Q_{1}-\frac{1}{3}\left(P_{1}-1\right)-\frac{\sigma}{3}\left(P_{1}-1\right)^{2} .
\end{aligned}
$$

If the universe expands without limit, $a \rightarrow \infty, t \rightarrow \infty$, then Eq. (42) is always satisfied, and $P_{1}, Q_{1}$ are the asymptotic values of $P, Q$. For $\mathcal{C}>0$, the variable $P$ plays the role of a time parameter, since $P(a)$ is monotonically decreasing, with $P>P_{1}$. Thus, in $(P, Q)$-space, the cosmological evolution is determined by the curve

$$
Q(P)=Q_{1}+\frac{P-P_{1}}{3}+\frac{\sigma}{3}\left[(P-1)^{2}+\left(P_{1}-1\right)^{2}\right] .
$$

There is a well-defined cosmological evolution when this curve passes through the region defined by the inequalities (27) and (28), which in turn depends on the values of the parameters $P_{1}, Q_{1}$ and $\sigma$. A discussion analogous to the previous one is also valid for $\mathcal{C}=0$.

One can verify that for $\mathcal{C} \geq 0$ there is a region of parameter space for which $f$ is positive. The solutions with $0<f_{0}<\infty$ represent models that avoid a cosmological singularity (in density and curvature), and undergo accelerated expansion from $a_{0}$. Furthermore, for $\mathcal{C}>0$, there are solutions which have infinite acceleration at $a_{0}$. Indeed, when the curve Eq. (46) crosses the critical curve

$$
Q_{c}(P)=\frac{2}{27}\left[9 P-8-(4-3 P)^{3 / 2}\right]
$$

which annihilates the denominator appearing in Eq. (41), then, there is infinite acceleration or deceleration, $f_{0} \rightarrow$ $\pm \infty$. This occurs if and only if either $\sigma \leq 3 / 2,0<\tau<$ $(5-\sigma) / 27$, or $\sigma>3 / 2,0<\tau<\left(16 \sigma^{2}-12 \sigma+3\right) / 48 \sigma^{3}$. A sufficient condition for the solutions with the + sign in Eq. (29) to have infinite acceleration $f_{0}$ is $1 / 10<\sigma<$ $1 / 2,0<\tau<(10 \sigma-1) / 12 \sigma(2 \sigma+3)$. These conditions on $\sigma$ and $\tau$ have non-empty intersection. The first condition translates into bounds on the density in terms of the bulk black hole mass and curvature coupling parameter:

$$
\frac{9}{80} \beta \mathcal{C}<\kappa_{4}^{2} \rho_{0}<\frac{9}{16} \beta \mathcal{C}
$$

The bulk black hole is crucial to the possibility of infinite acceleration. For $\mathcal{C}=0$, one can show that $f_{0}$ cannot become $+\infty$ for a radiation era. We also note that all the above results hold independently of the spatial curvature $k$ of the universe.

The accelerating expansion at and near $a_{0}$, that is driven by geometric effects, serves as a "geometric" form of inflation, very different from conventional scalar field inflation. This could be interpreted as an alternative to inflaton scenarios, based on a quantum-gravity correction. However, there remain two crucial caveats. 
(1) For $k \geq 0$, there is no exit from acceleration for the range of accelerating parameters $\sigma, \tau$ which give infinite $f_{0}$, in the radiation era. This can be seen, using Eq. (29), from the fact that the sum of the first two terms in Eq. (40) is always positive, since $P^{2}-6 Q$ is monotonically decreasing, and the last term in Eq. (40), proportional to $\dot{\Theta}$, is also positive. The range of $\sigma, \tau$ values gives only a sufficient condition for acceleration, and we have not been able to characterize the whole parametric space $\left(P_{1}, Q_{1}, \sigma, \beta\right)$. Therefore, it is still possible that some parameters exist that lead to an exit from inflation.

(2) Those solutions with $f_{0} \rightarrow \infty$ have a divergence of the Ricci scalar $R$ on the brane, even though the density is finite. This is impossible in general relativity or the Randall-Sundrum model, since in both cases $R=-T$, where $T$ is the trace of the brane energy momentum tensor. This simple relation breaks down when there are curvature corrections, and the bulk curvature, interacting with the brane curvature and matter, plays a decisive role. Thus, the minimal epoch $a_{0}$ marks a curvature singularity, and the brane spacetime geometry breaks down there.

It is also worth reiterating that besides the solutions with positive $f_{0}$, there are solutions that are always decelerating.

The acceleration-deceleration behaviour of the pure Gauss-Bonnet and the pure induced gravity models, is very different. For the Gauss-Bonnet case, we find from Eq. (15) that for $\mathcal{C}>0$ it is

$$
f=-\frac{1}{4 \alpha}+\frac{1}{16 \alpha}\left(1-\frac{64 I^{2}}{J^{2}}\right)\left(2 J+\frac{\dot{J}}{H}\right)+\frac{16 \tilde{c}_{2}}{\alpha J},
$$

where

$$
\begin{aligned}
& \sqrt{J} \frac{\dot{J}}{H}=-\frac{2 \tilde{\sigma}(1+w)\left(I^{2}-\tilde{c}_{2}\right)^{3(1+w) / 4}}{\sqrt{\left[\tilde{c}_{1}+\tilde{\sigma}\left(I^{2}-\tilde{c}_{2}\right)^{3(1+w) / 4}\right]^{2}+(8 I)^{3}}} \times \\
& \quad \times\left[J^{3 / 2}+\frac{512 I}{\tilde{\sigma}(1+w)}\left(I^{2}-\tilde{c}_{2}\right)^{(1-3 w) / 4}\right],
\end{aligned}
$$

with $\tilde{c}_{1}=\sqrt{\alpha} \kappa_{5}^{2} \lambda / \sqrt{2}, \tilde{c}_{2}=\left(3+4 \alpha \Lambda_{5}\right) / 192$, and $\tilde{\sigma}=$ $\left(\sqrt{\alpha} \kappa_{5}^{2} \rho_{0} / \sqrt{2}\right)(8 / \alpha \mathcal{C})^{3(1+w) / 4}$. In $(I, J)$-space, the curve defining the evolution of the Gauss-Bonnet universe is

$$
\begin{aligned}
& J(I)=\left\{\tilde{c}_{1}+\tilde{\sigma}\left(I^{2}-\tilde{c}_{2}\right)^{3(1+w) / 4}\right. \\
& \left.+\sqrt{\left[\tilde{c}_{1}+\tilde{\sigma}\left(I^{2}-\tilde{c}_{2}\right)^{3(1+w) / 4}\right]^{2}+(8 I)^{3}}\right\}^{2 / 3}
\end{aligned}
$$

Here, $I$ can play the role of time parameter, with $I(a)$ monotonically decreasing for $\mathcal{C}>0$. In the case $\mathcal{C}=0$, similar expressions are valid. The only candidate quantity in Eq. (49) for producing divergence in $f$ is the term $2 J+\dot{J} / H$. By carefully examining the various situations, we obtain the result that in the radiation era of the Gauss-Bonnet universe there is no infinite acceleration. In the combined theory, because there is no infinitedensity regime, the early universe behaviour cannot be obtained by expanding for large $\rho$. On the contrary, in the pure Gauss-Bonnet theory, equation (33) is the large $\rho$ expansion, from which we can see furthermore that there is no initial acceleration, and for $\rho \rightarrow \infty, R \rightarrow+\infty$.

Finally, the induced gravity equation (19) gives in the radiation era:

$$
\begin{aligned}
& f(\rho)=\frac{\kappa_{4}^{2}}{3}(\lambda-\rho)+\frac{2}{r^{2}} \\
& \pm \frac{\sqrt{2}}{\sqrt{3} r}\left(2 \kappa_{4}^{2} \lambda-\Lambda_{5}+\frac{6}{r^{2}}\right) \times \\
& \times\left\{2 \kappa_{4}^{2}\left[\lambda+\left(1-\frac{3 \mathcal{C}}{\kappa_{4}^{2} \rho_{0}}\right) \rho\right]-\Lambda_{5}+\frac{6}{r^{2}}\right\}^{-1 / 2}
\end{aligned}
$$

We see from Eqs. (19) and (52) that among the solutions of the induced gravity model, there are some which start with initial singularity $a=0$, as in the conventional model with $f=-\infty$. Moreover, there is at least one family of solutions for the branch with the + sign, characterized by the conditions $2 \kappa_{4}^{2} \lambda-\Lambda_{5}+6 / r^{2}>0$, $\kappa_{4}^{2} \rho_{0}<3 \mathcal{C}$ and $k \leq 0$, which start at a finite scale factor with infinite acceleration, qualitatively similar to our model. Adopting the point of view that the characteristics of infinite-density avoidance and initial infinite acceleration are interesting cosmological features which are still present in the combined induced gravity plus GaussBonnet model, we can say that the inclusion of the GaussBonnet term has improved the situation by eliminating all the infinite-density solutions.

\section{Late universe}

For the parameters that allow $a \rightarrow \infty$, Eq. (29) is approximated as

$$
H^{2}+\frac{k}{a^{2}} \approx \frac{4-3 \beta-\gamma}{12 \beta \alpha}+\nu \kappa_{4}^{2} \rho,
$$

neglecting terms $O\left(\rho^{4 / 3}\right)$, where the dimensionless parameters $\gamma$ and $\nu$ are

$$
\begin{aligned}
\gamma= & 8 \sqrt{P_{1}^{2}-6 Q_{1}} \cos \left(\Theta_{1} \pm \frac{\pi}{3}\right), \\
\nu= & \frac{2}{3 \sqrt{P_{1}^{2}-6 Q_{1}}}\left[\cos \left(\Theta_{1} \pm \frac{\pi}{3}\right)+\sin \left(\Theta_{1} \pm \frac{\pi}{3}\right) \times\right. \\
& \left.\times \frac{3 Q_{1}+2 P_{1}\left(1-P_{1}\right)}{\sqrt{3} \sqrt{4 Q_{1}\left(9 P_{1}-8\right)+4 P_{1}^{2}\left(1-P_{1}\right)-27 Q_{1}^{2}}}\right],(55)
\end{aligned}
$$

with $\Theta_{1}=\Theta\left(P_{1}, Q_{1}\right)$.

First, we observe that the bulk black hole mass $\mathcal{C}$ does not appear, which means that even if it is non-zero, it decouples during the cosmological evolution and does not 
affect the late universe dynamics. The bulk is felt in the late universe only through its vacuum energy $\Lambda_{5}$.

Second, for the branch with the + sign in Eq. (29), because of the inequalities (27), (28), it follows that $\nu>0$. Thus, although the last term in Eq. 29] is negative, in the late-time limit it produces both a negative cosmological constant, $-\gamma$, which contributes to the total cosmological constant, and a linear $\rho$ term with positive Newton constant. For the branch with the - sign, $\nu$ may be negative. Third, it is seen from the $a \rightarrow \infty$ limit of Eq. (29) that the quantity $4-3 \beta-\gamma$ is always non-negative. Therefore, the conventional cosmology is recovered with positive effective gravitational and cosmological constants:

$$
G_{\mathrm{eff}}=3 \nu G_{4}, \quad \Lambda_{\mathrm{eff}}=\frac{4-3 \beta-\gamma}{4 \beta \alpha} .
$$

In the Gauss-Bonnet case, the late-universe limit of Eq. (15) is

$$
H^{2}+\frac{k}{a^{2}} \approx \frac{\Lambda_{\mathrm{eff}}}{3}+\frac{8 \pi G_{\mathrm{eff}}}{3} \rho,
$$

neglecting terms $O\left(\rho^{4 / 3}\right)$, where the effective constants are

$$
\begin{aligned}
\Lambda_{\mathrm{eff}} & =\frac{3}{8 \alpha}\left(-2+\frac{64 I_{1}^{2}}{J_{1}}+J_{1}\right) \\
G_{\mathrm{eff}} & =\frac{G_{5}}{2 \sqrt{2 \alpha}} \sqrt{J_{1}}\left[\frac{J_{1}^{2}-\left(8 I_{1}\right)^{2}}{J_{1}^{3}+\left(8 I_{1}\right)^{3}}\right] .
\end{aligned}
$$

Here $I_{1}, J_{1}$ are the asymptotic values for $a \rightarrow \infty$ of the variables $I, J$, defined in terms of the parameters $\tilde{c}_{1}, \tilde{c}_{2}$ of the Gauss-Bonnet model by the relations $I_{1}=\sqrt{\tilde{c}_{2}}$, $J_{1}=\left[\tilde{c}_{1}+\sqrt{\tilde{c}_{1}^{2}+\left(64 \tilde{c}_{2}\right)^{3 / 2}}\right]^{2 / 3}$. The previous remarks concerning the non-appearance of $\mathcal{C}$ in the above equations, as well as the positivity of the effective Newton and cosmological constants, are still valid.

When the brane tension is zero, the Friedmann equation (53) recovers the standard general relativity behaviour, since the coefficient $\nu$ in Newton's constant remains positive and nonzero if we set $\lambda=0$. Therefore, if both curvature corrections are combined, the conventional cosmology is recovered, even for a tensionless brane. On the contrary, in the pure Gauss-Bonnet equation (57), the brane tension is essential, since $\lambda=0$ implies $G_{\text {eff }}=0$. This is like the pure Randall-Sundrum case, where positive brane tension is necessary in order to recover the standard Friedmann equation [2, 20].

The late-time limit of the pure induced gravity Friedmann equation (19) gives the positive constants

$$
\begin{aligned}
& \Lambda_{\text {eff }}=\kappa_{4}^{2} \lambda+\frac{6}{r^{2}} \pm \frac{\sqrt{6}}{r^{2}} \sqrt{\left(2 \kappa_{4}^{2} \lambda-\Lambda_{5}\right) r^{2}+6} \\
& G_{\text {eff }}=G_{4}\left[1 \pm\left\{\frac{r^{2}}{6}\left(2 \kappa_{4}^{2} \lambda-\Lambda_{5}\right)+1\right\}^{-1 / 2}\right]
\end{aligned}
$$

When there is no brane tension, and even no bulk cosmological constant, general relativity is still recovered [5].

\section{CONCLUSIONS}

We studied the cosmology of a brane-world with curvature corrections to the Randall-Sundrum gravitational action, i.e. a four-dimensional curvature term of induced gravity and a five-dimensional Gauss-Bonnet term. The fundamental parameters appearing in the model are: three energy scales, i.e. the fundamental Planck mass $M_{5}$, the induced-gravity crossover energy scale $r^{-1}$, and the Gauss-Bonnet coupling energy scale $\alpha^{-1 / 2}$, and two vacuum energies, i.e. the bulk cosmological constant $\Lambda_{5}$ and the brane tension $\lambda$. These parameters determine the cosmological evolution of the brane universe.

We derived the Friedmann equation of the combined curvature effects, Eq. (29), which smoothly matches to the induced gravity equation when the Gauss-Bonnet term vanishes. This equation has a structure which is quite different from its two limiting forms. All the solutions of the cosmological model are of finite density, independently of the spatial curvature of the universe and the equation of state. This is remarkable, since the Gauss-Bonnet correction on its own dominates at early times and does not remove the infinite-density singularity, while the induced gravity correction on its own mostly affects the late-time evolution. However, the combination of these curvature corrections produces an "interaction" that is not obviously the superposition of their separate effects. In general terms, the early-universe behaviour is strongly modified by the effective coupling of the $5 \mathrm{D}$ curvature to the matter.

The late cosmological evolution of our model follows the standard cosmology, even for zero brane tension, with a positive Newton constant for one of the two branches of the solutions and positive cosmological constant.

We also showed that a radiation brane can, for some parameter values, undergo accelerated expansion at and near the minimal scale factor, independently of the spatial curvature of the universe. When there is a black hole in the bulk, a subset of these solutions has infinite acceleration at $a_{0}$, which signals the "birth" of an accelerated universe at finite energy, but with a curvature singularity.

\section{Acknowlegements}

We thank C. Barcelo, N. Deruelle, C. Germani, A. Kehagias, J. Lidsey and P. Singh for valuable discussions and comments. G.K. acknowledges partial support from FONDECYT grant 3020031 and from Empresas CMPC. Centro de Estudios Científicos is a Millennium Science Institute and is funded in part by grants from Fundacion Andes and the Tinker Foundation. R.M. is supported by PPARC. E.P. is partially supported by the NTUA research program "Thalis". 
[1] L. Randall and R. Sundrum, Phys. Rev. Lett. 83, 4690 (1999) hep-th/9906064.

[2] P. Binétruy, C. Deffayet, U. Ellwanger and D. Langlois, Phys. Lett. 477B, 285 (2000) hep-th/9910219.

[3] C. Csaki, M. Graesser, C. Kolda and J. Terning, Phys. Lett. 462B, 34 (1999) hep-ph/9906513; J. Cline, C. Grojean and G. Servant, Phys. Rev. Lett. 83, 4245 (1999) hep-ph/9906523.

[4] H. Collins and B. Holdom, Phys. Rev. D62, 105009 (2000) hep-ph/0003173.

[5] G. Dvali, G. Gabadadze and M. Porati, Phys. Lett. 485B, 208 (2000) hep-th/0005016; G. Dvali and G. Gabadadze, Phys. Rev. D63, 065007 (2001) hep-th/0008054.

[6] D. Lovelock, J. Math. Phys. 12, 498 (1971).

[7] B. Zwiebach, Phys. Lett. 156B, 315 (1985); B. Zumino, Phys. Rept. 137, 109 (1986); D.J. Gross and J.H. Sloan, Nucl. Phys. B291, 41 (1987).

[8] Y. Shtanov, hep-th/0005193 S. Nojiri and S.D. Odintsov, JHEP 07, 049 (2000) hep-th/0006232; C. Deffayet, Phys. Lett. 502B, 199 (2001) hep-th/0010186; G. Kofinas, JHEP 08, 034 (2001) hep-th/0108013; N.J. Kim, H.W. Lee and Y.S. Myung, Phys. Lett. 504B, 323 (2001) hep-th/0101091; C. Deffayet, G. Dvali and G. Gabadadze, Phys. Rev. D65, 044023 (2002) astro-ph/0105068; C. Deffayet, S.J. Landau, J. Raux, M. Zaldarriaga and P. Astier, Phys. Rev. D66, 024019 (2002) astro-ph/0201164.

[9] E. Kiritsis, N. Tetradis and T.N. Tomaras, JHEP 03, 019 (2002) hep-th/0202037.

[10] V. Sahni and Y. Shtanov, Int. J. Mod. Phys. 11, 1 (2002) gr-qc/0205111; U. Alam and V. Sahni, astro-ph/0209443

[11] K. Maeda, S. Mizuno and T. Torii, Phys. Rev. D68, 024033 (2003) gr-qc/0303039.

[12] G. Kofinas, E. Papantonopoulos and I. Pappa, Phys. Rev. D66, 104014 (2002) hep-th/0112019; G. Kofinas, E. Papantonopoulos and V. Zamarias, Phys. Rev. D66, 104028 (2002) hep-th/0208207; A. Lue and G. Starkman, Phys. Rev. D67, 064002 (2003) astro-ph/0212083; G. Dvali, A. Gruzinov and M. Zaldarriaga, Phys. Rev. D68, 024012 (2003) hep-ph/0212069.

[13] J.E. Kim, B. Kyae and H.M. Lee, Nucl. Phys. B582, 296 (2000) hep-th/0004005; J.E. Kim and H.M. Lee, Nucl. Phys. B602, 346 (2001) hep-th/0010093; S. Nojiri and S.D. Odintsov, Phys. Lett. 493B, 153 (2000) hep-th/0007205; S. Nojiri, S.D. Odintsov and S. Ogushi, Phys. Rev. D65, 023521 (2002) hep-th/0108172; I.P. Neupane, JHEP 09, 040 (2000) hep-th/0008190; I.P. Neupane, Class. Quant. Grav. 19, 5507 (2002) hep-th/0106100; K.A. Meissner and M. Olechowski,
Phys. Rev. Lett. 86, 3708 (2001) hep-th/0009122; K.A. Meissner and M. Olechowski, Phys. Rev. D65, 064017 (2002) hep-th/0106203 ; E. Kiritsis, N. Tetradis and T. Tomaras, JHEP 0108, 012 (2001) hep-th/0106050.

[14] N. Deruelle and T. Dolezel, Phys. Rev. D62, 103502 (2000) gr-qc/0004021; B. Abdesselam and N. Mohammedi, Phys. Rev. D65, 084018 (2002) hep-th/0110143.

[15] C. Germani and C. Sopuerta, Phys. Rev. Lett. 88, 231101 (2002) hep-th/0202060.

[16] C. Charmousis and J. Dufaux, Class. Quantum Grav. 19, 4671 (2002) hep-th/0202107.

[17] I. Low and A. Zee, Nucl. Phys. B585, 395 (2000) hep-th/0004124; J.E. Lidsey, S. Nojiri and S. Odintsov, JHEP 06, 026 (2002) hep-th/0202198; P. Binetruy, C. Charmousis, S.C. Davis and J-F. Dufaux, Phys. Lett. 544B, 183 (2002) hep-th/0206089; J.P. Gregory and A. Padilla, hep-th/0304250 N. Deruelle and M. Sasaki, gr-qc/0306032 C. Barcelo, C. Germani and C.F. Sopuerta, gr-qc/0306072

[18] J.E. Lidsey and N.J. Nunes, Phys. Rev. D67, 103510 (2003) astro-ph/0303168.

[19] M.A. Luty, M. Porrati and M. Manera, hep-th/0303116 V.A. Rubakov, hep-th/0303125

[20] T. Shiromizu, K. Maeda and M. Sasaki, Phys. Rev. D62, 024012 (2000) gr-qc/9910076.

[21] D.G. Boulware and S. Deser, Phys. Rev. Lett. 55, 2656 (1985); J.T. Wheeler, Nucl. Phys. B268, 737 (1986); N. Deruelle and J. Madore, Phys. Lett. 186B, 25 (1987); R.C. Myers, gr-qc/9811042 R-G. Cai, Phys. Rev. D65, 084014 (2002) hep-th/0109133.

[22] S. Davis, Phys. Rev. D67, $024030 \quad$ (2003) hep-th/0208205; E. Gravannis and S. Willison, Phys. Lett. 562B, 118 (2003) hep-th/0209076; N.E. Mavromatos and J. Rizos, Phys. Rev. D62, 124004 (2000) hep-th/0008074; Y.M. Cho, I.P. Neupane and P.S. Wesson, Nucl. Phys. B621, 388 (2002) hep-th/0104227.

[23] J. Zanelli, hep-th/0206169

[24] J. Chrisostomo, R. Troncoso and J. Zanelli, Phys. Rev. D62, 084013 (2000) hep-th/0003271.

[25] S.O. Alexeyev, A.V. Toporensky and V.O. Ustiansky, gr-qc/9912071 S.O. Alexeyev, A.V. Toporensky and V.O. Ustiansky, Phys. Lett. 509B, 151 (2001) gr-qc/0009020; A. Toporensky and S. Tsujikawa, Phys. Rev. D65, 123509 (2002) gr-qc/0202067.

[26] R. Maartens, D. Wands, B.A. Bassett and I.P.C. Heard, Phys. Rev. D62, 041301 (2000) hep-ph/9912464.

[27] In taking the limit $\alpha \rightarrow 0$, one has to express $\Theta(P, Q)$ in terms of arctan, which is defined in the neighborhood of $+\infty$, instead of arccos which is not defined at $+\infty$. 